\title{
Unparticle Searches Through Compton Scattering
}

\author{
O. Çakıı* \\ Department of Physics, Ankara University, 06100 Tandogan, Ankara, Turkey \\ K. O. Ozansoy \\ Department of Physics, University of Wisconsin, Madison, WI 53706, USA and \\ Department of Physics, Ankara University, 06100 Tandogan, Ankara, Turkey
}

(Dated: November 19, 2018)

\begin{abstract}
We investigate the effects of unparticles on Compton scattering, $e \gamma \rightarrow e \gamma$ based on a future $e^{+} e^{-}$linear collider such as the CLIC. For different polarization configurations, we calculate the lower limits of the unparticle energy scale $\Lambda_{\mathcal{U}}$ for a discovery reach at the center of mass energies $\sqrt{s}=0.5 \mathrm{TeV}-3.0 \mathrm{TeV}$. It is shown that, especially, for smaller values of the mass dimension $d$, $(1<d<1.3)$, and for high energies and luminosities of the collider these bounds are very significant. As a stringent limit, we find $\Lambda_{\mathcal{U}}>80 \mathrm{TeV}$ for $d<1.3$ at $\sqrt{s}=3 \mathrm{TeV}$, and $1 \mathrm{ab}^{-1}$ integrated luminosity per year, which is comparable with the limits calculated from the other low and high energy physics implications.
\end{abstract}

*Electronic address: ocakir@science.ankara.edu.tr

${ }^{\dagger}$ Electronic address: oozansoy@physics.wisc.edu 


\section{INTRODUCTION}

Recently, Georgi proposed that the possibility of new physics effects of a hidden scale invariant sector living at a very high energy scale[1]. If such a scale invariant sector really exists then one can expect to observe the effects of it on our low energy physical phenomena. A very appealing feature of Georgi's unparticle formulation for such a scale invariant sector is that one can investigate the implications of it on the current low and high energy physics data. Considering the idea of [2], in [1], the scale invariant sector is presented by a set of the Banks-Zaks operators $\mathcal{O}_{B Z}$, and defined at the very high energy scale. Interactions of $\mathcal{O}_{B Z}$ and the Standard Model(SM) operators $\mathcal{O}_{S M}$ are expressed by the exchange of particles with a very high energy mass scale $\mathcal{M}_{\mathcal{U}}^{k}$ in the following form

$$
\frac{1}{\mathcal{M}_{\mathcal{U}}^{k}} O_{B Z} O_{S M}
$$

where BZ, and SM operators are defined as $O_{B Z} \in \mathcal{O}_{B Z}$ with mass dimension $d_{B Z}$, and $O_{S M} \in \mathcal{O}_{S M}$ with mass dimension $d_{S M}$. Low energy effects of the scale invariant $\mathcal{O}_{B Z}$ fields imply a dimensional transmutation. Thus, after the dimensional transmutation Eq.(11) is given as

$$
\frac{C_{\mathcal{U}} \Lambda_{\mathcal{U}}^{d_{B Z}-d}}{\mathcal{M}_{\mathcal{U}}^{k}} O_{\mathcal{U}} O_{S M}
$$

where $d$ is the mass dimension of the unparticle operator $O_{\mathcal{U}}$ (in Ref. [1], $d=d_{\mathcal{U}}$ ), and the constant $C_{\mathcal{U}}$ is a coefficient function.

In Refs. [1] and [3] main ideas for effective interactions between SM fields and the unparticles have been presented by Georgi. In [4], the collider phenomenology of unparticle physics has been explored in a great detail, and Feynman rules for spin 0, spin 1, or spin 2 unparticles coupled to a variety of SM gauge invariant operators have been explicitly given. Subsequently, many analysis on unparticles have been done for possible signatures and for limits from the collider experiments, astrophysical, and cosmology implications Ref. [5].

Searching for the new physics effects, the $e^{+} e^{-}$linear colliders have an exceptional advantageous for its appealing clean background, and the possibility for the options of $e \gamma$, and $\gamma \gamma$ colliders based on it. Recently, for the new physics searches, as a multi TeV energy electron-positron linear collider, the CLIC is seriously taken into account, and there are numerous works on the phenomenology potential of it. For the multi-TeV linear electron positron colliders and the physical potential of CLIC one can consult [7]. The CLIC $e^{+} e^{-}$ linear collider would also have the options for $e^{-} e^{-}, e \gamma$, and $\gamma \gamma$ collider options, and possibilities of polarized $e^{+}, e^{-}$beams. The $e \gamma$ option of a linear electron-positron collider, is also a favorable tool for searching new physics effects for example [9, 10, 11]. Namely, the center of mass energy of the $e \gamma$ collision can reach more than $90 \%$ of main $e^{+} e^{-}$center of mass energy. And also luminosity can be comparable with that of the main $e^{+} e^{-}$collision. In [8], a detailed analysis on $e \gamma$ option of an $e^{+} e^{-}$collider has been given.

Here, we consider the CLIC based $e \gamma$ collider to search for the unparticle physics effects, our results can easily be extended for other possible future $\mathrm{TeV}$-scale linear electron-positron colliders. Our calculations show that the unpolarized, and polarized e $\gamma$ collisions at $\mathrm{TeV}$ scale energies, and high luminosities are very sensitive for the unparticle searches. 


\section{COMPTON SCATTERING}

The Standard Model scattering amplitude for the Compton scattering is given as

$$
M_{S M}=M_{S M}^{s}+M_{S M}^{u}
$$

where

$$
\begin{aligned}
& M_{S M}^{s}=-\frac{e^{2}}{2 p_{1} \cdot k_{1}}\left[\bar{e}\left(p_{2}\right) \epsilon^{*}\left(k_{2}\right) \epsilon\left(k_{1}\right) \not k_{1} e\left(p_{1}\right)\right] \\
& \left.M_{S M}^{u}=-\frac{e^{2}}{2 p_{1} \cdot k_{2}}\left[\left[\bar{e}\left(p_{2}\right) \epsilon\left(k_{1}\right) e\left(p_{1}\right)\right]\left(p_{1} \cdot \epsilon_{2}^{*}\right)\right]+\left[\bar{e}\left(p_{2}\right) \epsilon\left(k_{1}\right) \epsilon^{*}\left(k_{2}\right) \not k_{2} e\left(p_{1}\right)\right]\right]
\end{aligned}
$$

where $\epsilon\left(k_{1}\right)\left(\epsilon\left(k_{2}\right)\right)$ is the polarization vector of the incoming(outgoing) photon to the vertex, $k_{1}\left(k_{2}\right)$, and $p_{1}\left(p_{2}\right)$ are the momenta of the incoming(outgoing) photon and electron. Therefore, using the center of mass reference frame kinematical relations relevant for the process $e^{-} \gamma \rightarrow e^{-} \gamma$ we get

$$
\left|M_{S M}\right|^{2}=-2 e^{4}\left[\frac{s}{u}+\frac{u}{s}\right]
$$

where, considering the high energy limits, we take the electron mass to be zero.

Interactions of the scalar unparticles with the electrons, and photons are given by [3, 44]. Therefore, the contribution to the scattering amplitude from the exchange of the scalar unparticle takes the form

$$
M^{t}{ }_{\mathcal{U}_{S}}=\frac{f(d)}{\Lambda_{\mathcal{U}}^{2 d-1}}\left[\bar{e}\left(p_{2}\right) e\left(p_{1}\right)\right]\left[\left(\epsilon_{2}^{*} \cdot \epsilon_{1}\right)\left(k_{1} \cdot k_{2}\right)-\left(\epsilon_{2}^{*} \cdot k_{1}\right)\left(\epsilon_{1} \cdot k_{2}\right)\right]\left[-q^{2}-i \epsilon\right]^{d-2}
$$

where

$$
f(d)=\frac{2 \lambda_{0}^{2} A_{d}}{\sin (d \pi)}, \quad A_{d}=\frac{16 \pi^{5 / 2}}{(2 \pi)^{2 d}} \frac{\Gamma(d+1 / 2)}{\Gamma(d-1) \Gamma(2 d)} .
$$

In principle, the coupling $\lambda_{\gamma 0}$ of the scalar unparticle to the photons can be different than the coupling $\lambda_{e 0}$ to the electrons. Here, for simplicity, we assume $\lambda_{\gamma 0}=\lambda_{e 0}=\lambda_{0}$ without lost of generality. Thus, for the scalar unparticle exchange, one can find

$$
\left|M_{U_{S}}^{t}\right|^{2}=\frac{[f(d)]^{2}}{4 \Lambda_{\mathcal{U}}^{(4 d-2)}}[-t]^{2 d-1}
$$

In Figure 1, we plot a schematic view of effects of the scalar unparticles to the total unpolarized cross section with respect to the center of mass energy $\sqrt{s}=E_{C M}$ of $e^{+} e^{-}$collision. Plotting this figure, we assume the unparticle energy scale $\Lambda_{\mathcal{U}}=2 \mathrm{TeV}$, scalar unparticle coupling $\lambda_{0}=1$, and the center of mass frame scattering angle region $|\cos \theta|<0.9$. According to the Figure 1, one can see that, for the mass dimension values $d<1.3$ effects of the scalar unparticles are very significant.

In Figure 2, we present the unpolarized differential cross section depending on the scattering angle for the $\mathrm{SM}$ and $\mathrm{SM}+\mathcal{U}_{S}$ for $d=1.1,1.3$. We assume $\lambda_{0}=1$ and $\Lambda_{\mathcal{U}}=1 \mathrm{TeV}$ at the center of mass energy of $\sqrt{s}=3 \mathrm{TeV}$. One can notice that under these assumptions, the scalar unparticle effect on the differential cross section is very significant. 


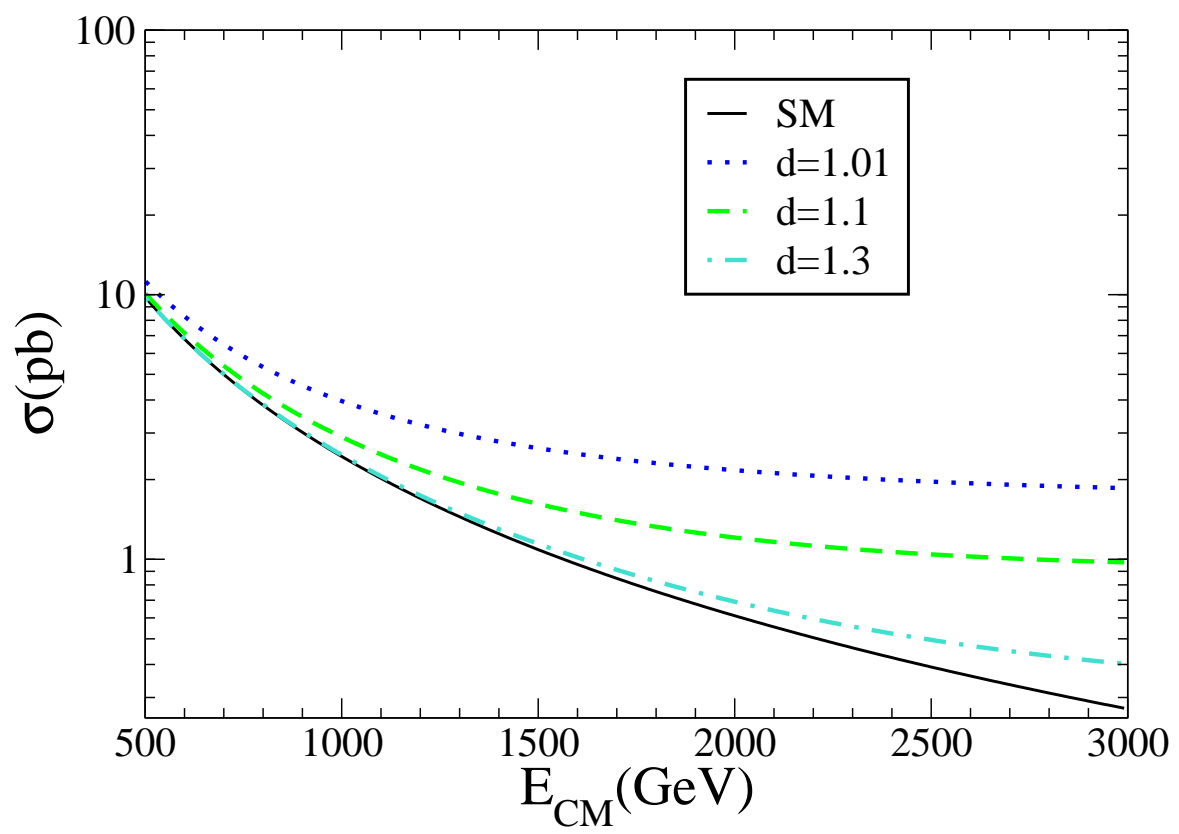

FIG. 1: Tot system for SN

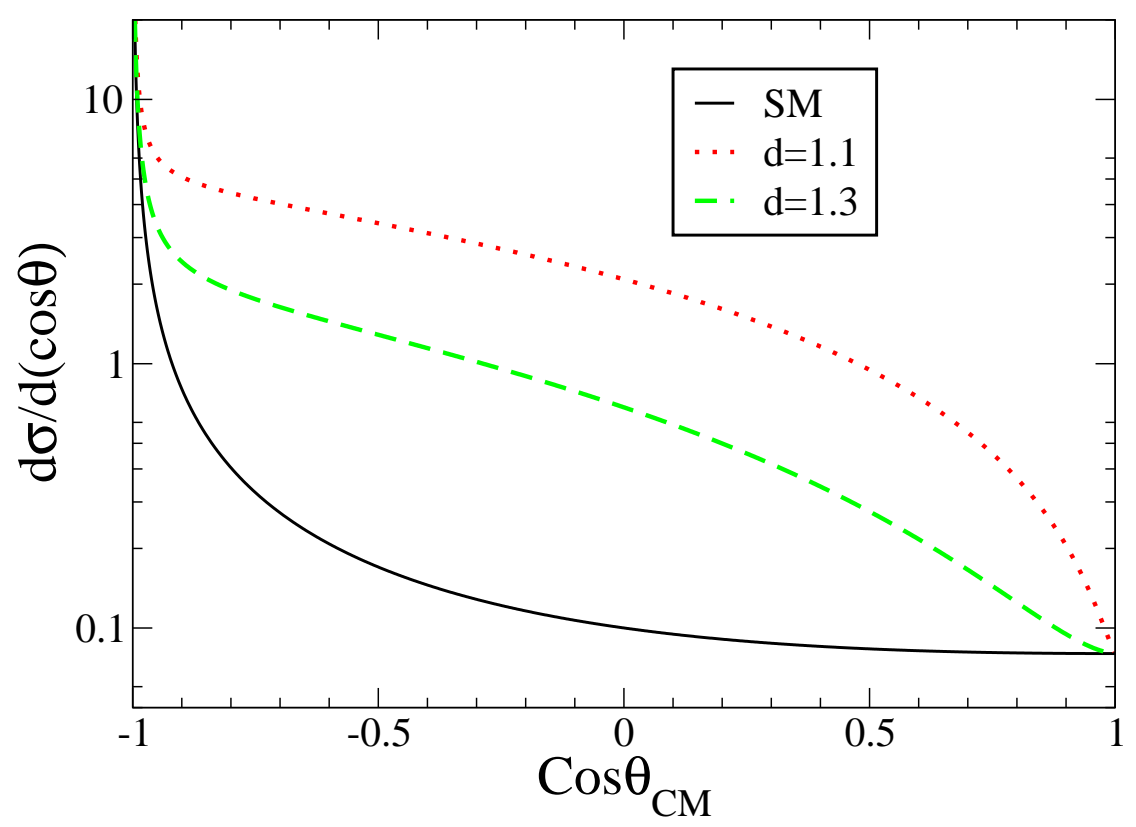

FIG. 2: The unpolarized differential cross section depending on the scattering angle for SM and $\mathrm{SM}+\mathcal{U}_{S}$ at $\sqrt{s}=3 \mathrm{TeV}$, where we take $\Lambda_{\mathcal{U}}=1 \mathrm{TeV}, \lambda_{0}=1$

\section{UNPARTICLE EFFECTS ON THE POLARIZED COMPTON SCATTERING}

Polarized cross section for the Compton scattering depends on the average helicity of the back-scattered photon $h_{\gamma}=h_{\gamma}\left(h_{0}, h_{e}\right)$ (where $h_{0}$ is the helicity of the laser photon, $h_{e}$ is the 
helicity of the initial electron), and the helicity $P$ of the electron can be written as

$$
\begin{aligned}
\frac{d \sigma}{d \cos \theta}= & \frac{1}{(32 \pi)} \int_{x_{\min }}^{0.83} d x \frac{f_{\gamma}(x)}{x s_{e e}} \\
& \times\left[\left(\frac{1+P h_{\gamma}(x)}{2}\right)\left|M^{S M+\mathcal{U}_{S}}(R R)\right|^{2}+\left(\frac{1-P h_{\gamma}(x)}{2}\right)\left|M^{S M+\mathcal{U}_{S}}(R L)\right|^{2}\right]
\end{aligned}
$$

where $\sqrt{\hat{s}}=\sqrt{x s}$ is the reduced center of mass energy of the back scattered photon beam, $\mathrm{x}$ is the energy fraction, $x_{\text {min }}=E_{0} E_{e} / m_{e}^{2}, E_{0}\left(E_{e}\right)$ is the energy of laser photon(initial electron) with respect to the laboratory frame, $m_{e}$ is the electron mass. We sum over the final helicities, therefore, $R, L$ stand for the initial helicities of the electron, and the photon beams. Also, the energy spectrum of the back scattered photon $f_{\gamma}(x)$ is given as follows [8]

$$
f_{\gamma}\left(x, h_{e}, h_{0}\right)=\frac{1}{g(\kappa)+h_{e} h_{0} y(\kappa)}\left[\frac{1}{1-x}+1-x-4 r(1-r)+h_{e} h_{0} r \kappa(1-2 r)(2-x)\right]
$$

where $r=x / \kappa(1-x), \kappa=4 E_{e} E_{0} / m_{e}^{2}$, and

$$
\begin{aligned}
& g(\kappa)=\left[1-\frac{4}{\kappa}-\frac{8}{\kappa^{2}}\right] \ln (\kappa+1)+\frac{1}{2}+\frac{8}{\kappa}-\frac{1}{2(\kappa+1)^{2}} \\
& y(\kappa)=\left[1+\frac{2}{\kappa}\right] \ln (\kappa+1)-\frac{5}{2}+\frac{1}{\kappa+1}-\frac{1}{2(\kappa+1)^{2}}
\end{aligned}
$$

The average helicity of the back-scattered photons is given [8]

$$
h_{\gamma}\left(x, h_{e}, h_{0}\right)=\frac{h_{0}(1-2 r)\left(1-x+\frac{1}{1-x}\right)+h_{e} r \kappa\left[1+(1-x)(1-2 r)^{2}\right]}{1-x+\frac{1}{1-x}-4 r(1-r)-h_{e} h_{0} r \kappa[(2 r-1)(2-x)]} .
$$

Using the above expressions, for different configurations of $\left(h_{e}, h_{0}, P\right)$, we plot the total cross section with respect to the center of mass energy $E_{C M}$. In Figure 3, and Figure 4, we plot a schematic view of effects of the scalar unparticles on the total polarized cross section for the polarization configurations $P_{1} \equiv\left(h_{e}=1, h_{0}=1, P=-1\right)$, and $P_{2} \equiv\left(h_{e}=\right.$ $\left.1, h_{0}=-1, P=-1\right)$, respectively. For the figures, we assume $\Lambda_{\mathcal{U}}=2 \mathrm{TeV}, \lambda_{0}=1$, and the center of mass frame scattering angle region $|\cos \theta|<0.9$. According to the Figure 3 , and 4, one can immediately notice that for the mass dimension values $d<1.3$, the effects of the scalar unparticles are considerably significant. And, also one can see that, due to the polarization dependence of the photon number density, the cross section values associated with the polarization configurations $P_{1}$ and $P_{2}$ are remarkably different.

\section{LIMITS AND DISCUSSIONS}

In the calculations, for the discovery reach of the energy scale of unparticles, we use the standard chi-square analysis for the following $\chi^{2}$ function

$$
\chi^{2}=\left(\frac{\mathcal{L}}{\sigma^{S M}}\right)\left[\sigma^{S M}-\sigma^{S M+\mathcal{U}}\left(\Lambda_{\mathcal{U}}\right)\right]^{2}
$$




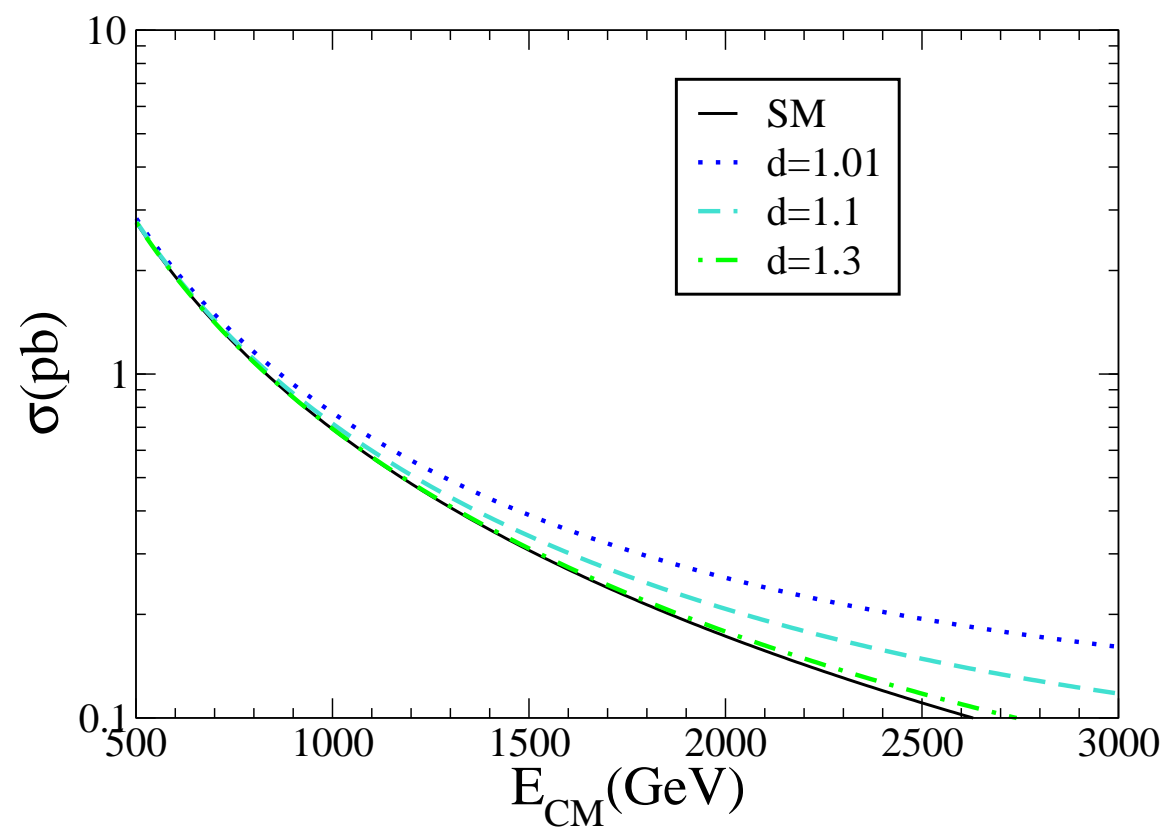

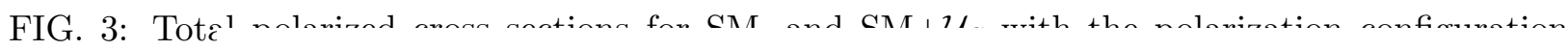
$P_{1} \equiv\left(h_{e}=1\right.$

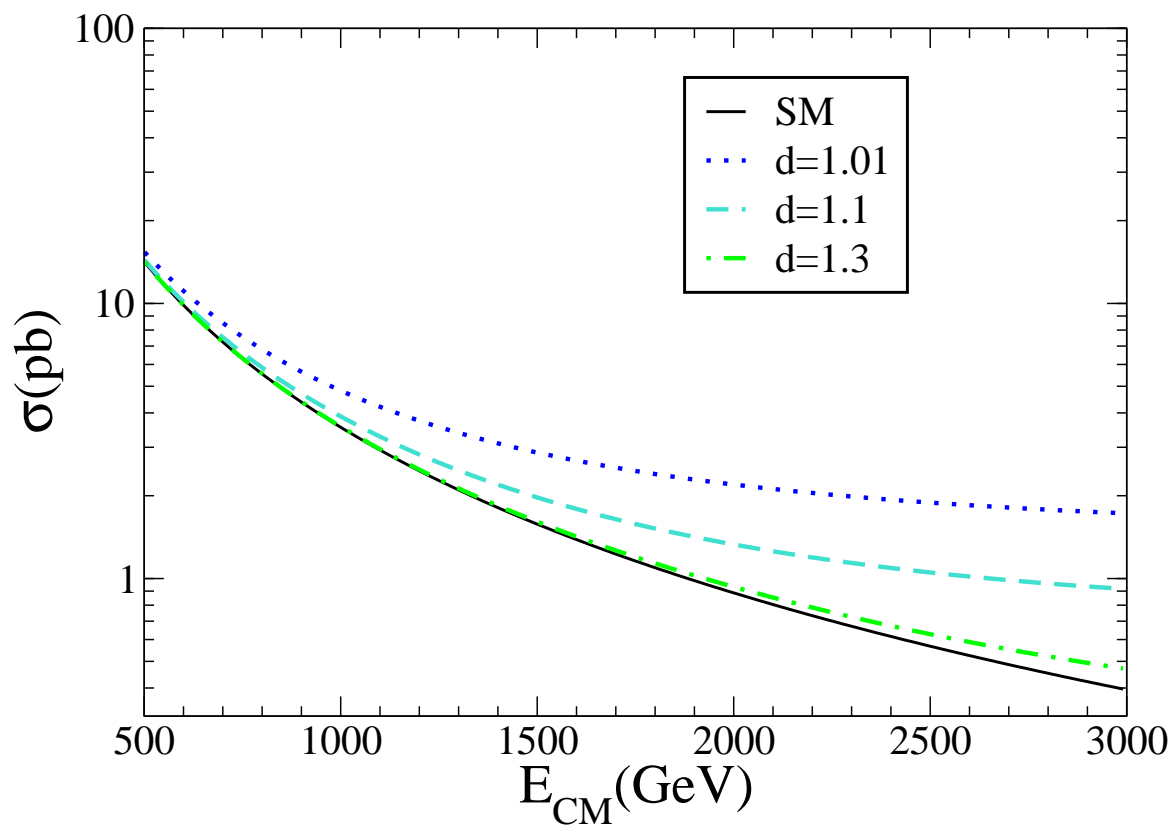

FIG. 4: Total polarized cross sections for $\mathrm{SM}$, and $\mathrm{SM}+\mathcal{U}_{S}$ with the polarization configuration $P_{2} \equiv\left(h_{e}=1, h_{0}=-1, P=-1\right)$.

For the one sided chi-square analysis we assume $\chi^{2} \geq 2.7$. And, to show that the luminosity dependence of the unparticle energy scale $\Lambda_{\mathcal{U}}$, we take two possible integrated luminosity values, $\mathcal{L}=100 \mathrm{fb}^{-1}$, and $\mathcal{L}=1000 \mathrm{fb}^{-1}$ per year. We observe that due to the polarization dependence of the photon number density, the limits are considerably different for different polarization configurations. Here, we present the limits for the $P_{1}=\left(h_{e}=\right.$ 
TABLE I: Lower limits on the $\Lambda_{\mathcal{U}}$ in the units of $\mathrm{GeV}$; the first entries in the paranthesies are for $\mathcal{L}=100 \mathrm{fb}^{-1}$, and the second ones for $\mathcal{L}=1000 \mathrm{fb}^{-1}$

\begin{tabular}{lcccccc}
\hline \hline$\sqrt{s} \mathrm{GeV}$ & $\mathrm{d}=1.0001$ & $\mathrm{~d}=1.01$ & $\mathrm{~d}=1.1$ & $\mathrm{~d}=1.3$ & $\mathrm{~d}=1.5$ & $\mathrm{~d}=1.9$ \\
\hline 500 & $(20300,36100)$ & $(18000,31700)$ & $(7400,11900)$ & $(2100,3000)$ & $(1060,1400)$ & $(670,800)$ \\
1000 & $(28700,51200)$ & $(25700,45200)$ & $(11100,17900)$ & $(3450,4950)$ & $(1760,2400)$ & $(1160,1450)$ \\
1500 & $(35200,62600)$ & $(31600,55600)$ & $(14100,22700)$ & $(4500,6500)$ & $(2400,3200)$ & $(1650,2100)$ \\
2000 & $(40700,72300)$ & $(36600,64400)$ & $(16600,26800)$ & $(5520,7950)$ & $(2990,3950)$ & $(2100,2560)$ \\
2500 & $(45400,80700)$ & $(41000,72100)$ & $(18900,30500)$ & $(6450,9250)$ & $(3550,4700)$ & $(2550,3100)$ \\
3000 & $(49800,88500)$ & $(45000,79150)$ & $(21050,34000)$ & $(7300,10500)$ & $(4050,5400)$ & $(2950,3600)$ \\
\hline \hline
\end{tabular}

$\left.1, h_{0}=-1, P=-1\right)$ polarization configuration which gives the strongest limits comparing the other polarization configurations. Our results is given in the TABLE I. As a remark, our lower limits on $\Lambda_{\mathcal{U}}$ are extracted assuming $\lambda_{0}=1$. In a similar calculation, one can also find upper limits on $\lambda_{0}$ assuming a fixed value of $\Lambda_{\mathcal{U}}$ such as $\Lambda_{\mathcal{U}}=1000 \mathrm{GeV}$. For example, using our limits presented in the TABLE $\square$ for the values $d=1.5, \sqrt{s}=3000 \mathrm{GeV}$, and $\mathcal{L}=100(1000) \mathrm{fb}^{-1}$ one can find $\lambda_{0 \max }=0.49(0.43)$ which is consistent with the limits given in the literature [6].

In conclusion, we put lower limits on $\Lambda_{\mathcal{U}}$ assuming the scalar unparticle effects on the polarized cross section can be distinguished from the SM contribution at 95\%C.L. In our calculations we consider the multi-TeV CLIC electron-positron collider for the center of mass energies $\sqrt{s}=0.5 \mathrm{TeV}-3.0 \mathrm{TeV}$, and the luminosities $\mathcal{L}=100 \mathrm{fb}^{-1}$, and $\mathcal{L}=1000 \mathrm{fb}^{-1}$ for different polarizations of the initial electron, the laser photon, and the back-scattered photon beams. Our calculations show that the limits of $\Lambda_{\mathcal{U}}$ get stronger as one increases the luminosity and the center of mass energy of the collider. It is shown that, especially, for smaller values of the mass dimension $d,(1<d<1.3)$, these bounds are very significant. As a stringent limit, from TABLE I, we find $\Lambda_{\mathcal{U}}>80 \mathrm{TeV}$ for $d<1.3$ at $\sqrt{s}=3 \mathrm{TeV}$ and $1 a b^{-1}$ luminosity per year, which is comparable with the limits calculated from other low and high energy physics implications.

\section{ACKNOWLEDGMENTS}

It is a pleasure to thank B. Balantekin for many helpful conversations and discussions. KOO would like to thank to the members of the Nuclear Theory Group of University of Wisconsin for their hospitality, and acknowledges support through the Scientific and Technical Research Council (TUBITAK) BIDEP-2219 grant. The work of O. C. was supported in part by the State Planning Organization (DPT) under grant no DPT-2006K-120470 and in part by the Turkish Atomic Energy Authority (TAEA) under grant no VII-B.04.DPT.1.05.

[1] H. Georgi, Phys. Rev. Lett. 98, 221601 (2007) arXiv:hep-ph/0703260.

[2] T. Banks and A. Zaks, Nucl. Phys. B 196, 189 (1982).

[3] H. Georgi, Phys. Lett. B 650, 275 (2007) arXiv:0704.2457 [hep-ph]]. 
[4] K. Cheung, W. Y. Keung and T. C. Yuan, Phys. Rev. Lett. 99, 051803 (2007) arXiv:0704.2588 [hep-ph]; Phys. Rev. D 76, 055003 (2007) [arXiv:0706.3155 [hep-ph]].

[5] C. H. Chen and C. Q. Geng, Phys. Rev. D 76, 115003 (2007) arXiv:0705.0689 [hep-ph]]; C. H. Chen and C. Q. Geng, Phys. Rev. D 76, 036007 (2007) arXiv:0706.0850 [hep-ph]]; S. L. Chen and X. G. He, Phys. Rev. D 76, 091702 (2007) arXiv:0705.3946 [hep-ph]]; T. M. Aliev, A. S. Cornell and N. Gaur, Phys. Lett. B 657, 77 (2007) arXiv:0705.1326 [hep-ph]]; T. M. Aliev, A. S. Cornell and N. Gaur, JHEP 0707, 072 (2007) arXiv:0705.4542 [hep-ph]]; C. H. Chen and C. Q. Geng, arXiv:0709.0235 [hep-ph]; G. J. Ding and M. L. Yan, Phys. Rev. D 76, 075005 (2007) arXiv:0705.0794 [hep-ph]]; G. J. Ding and M. L. Yan, arXiv:0706.0325 [hep-ph]; X. Q. Li and Z. T. Wei, Phys. Lett. B 651, 380 (2007) arXiv:0705.1821 [hep-ph]]; X. Q. Li, Y. Liu and Z. T. Wei, arXiv:0707.2285 [hep-ph]; Y. Liao, Phys. Rev. D 76, 056006 (2007) arXiv:0705.0837 [hep-ph]]; Y. Liao and J. Y. Liu, Phys. Rev. Lett. 99, 191804 (2007) arXiv:0706.1284 [hep-ph]]; M. A. Stephanov, Phys. Rev. D 76, 035008 (2007) [arXiv:0705.3049 [hep-ph]]; P. J. Fox, A. Rajaraman and Y. Shirman, Phys. Rev. D 76, 075004 (2007) arXiv:0705.3092 [hep-ph]]; C. D. Lu, W. Wang and Y. M. Wang, Phys. Rev. D 76, 077701 (2007) arXiv:0705.2909 [hep-ph]]; N. Greiner, Phys. Lett. B 653, 75 (2007) arXiv:0705.3518 [hep-ph]]; D. Choudhury, D. K. Ghosh and Mamta, Phys. Lett. B 658, 148 (2008) arXiv:0705.3637 [hep-ph]]; H. Davoudiasl, Phys. Rev. Lett. 99, 141301 (2007) arXiv:0705.3636 [hep-ph]]; J. McDonald, arXiv:0709.2350 [hep-ph]; S. L. Chen, X. G. He and H. C. Tsai, JHEP 0711, 010 (2007) arXiv:0707.0187 [hep-ph]]; P. Mathews and V. Ravindran, Phys. Lett. B 657, 198 (2007) arXiv:0705.4599 [hep-ph]]; S. Zhou, arXiv:0706.0302 [hep-ph]; M. Bander, J. L. Feng, A. Rajaraman and Y. Shirman, Phys. Rev. D 76, 115002 (2007) arXiv:0706.2677 [hep-ph]]; T. G. Rizzo, JHEP 0710, 044 (2007) arXiv:0706.3025 [hepph]]; R. Mohanta and A. K. Giri, Phys. Rev. D 76, 075015 (2007) arXiv:0707.1234 [hep-ph]]; D. Choudhury and D. K. Ghosh, arXiv:0707.2074 [hep-ph]; R. Mohanta and A. K. Giri, Phys. Rev. D 76, 057701 (2007) arXiv:0707.3308 [hep-ph]]; E. O. Iltan, arXiv:0710.2677 [hep-ph]; N. V. Krasnikov, Int. J. Mod. Phys. A 22, 5117 (2007) arXiv:0707.1419 [hep-ph]]; A. Lenz, Phys. Rev. D 76, 065006 (2007) arXiv:0707.1535 [hep-ph]]; H. Zhang, C. S. Li and Z. Li, Phys. Rev. D 76, 116003 (2007) arXiv:0707.2132 [hep-ph]]; Y. Nakayama, Phys. Rev. D 76, 105009 (2007) arXiv:0707.2451 [hep-ph]]; N. G. Deshpande, X. G. He and J. Jiang, Phys. Lett. B 656, 91 (2007) arXiv:0707.2959 [hep-ph]]; A. Delgado, J. R. Espinosa and M. Quiros, JHEP 0710, 094 (2007) arXiv:0707.4309 [hep-ph]]; S. Hannestad, G. Raffelt and Y. Y. Y. Wong, Phys. Rev. D 76, 121701 (2007) arXiv:0708.1404 [hep-ph]]; P. K. Das, Phys. Rev. D 76, 123012 (2007) arXiv:0708.2812 [hep-ph]]; G. Bhattacharyya, D. Choudhury and D. K. Ghosh, Phys. Lett. B 655, 261 (2007) arXiv:0708.2835[hep-ph]]; A. T. Alan and N. K. Pak, arXiv:0708.3802 [hep-ph]; A. Freitas and D. Wyler, arXiv:0708.4339 [hep-ph]; T. i. Hur, P. Ko and X. H. Wu, Phys. Rev. D 76, 096008 (2007) arXiv:0709.0629 [hep-ph]]; A. Kobakhidze, Phys. Rev. D 76, 097701 (2007) arXiv:0709.3782 [hep-ph]]; G. j. Ding and M. L. Yan, arXiv:0709.3435 [hep-ph]; K. Huitu and S. K. Rai, arXiv:0711.4754 [hep-ph].

[6] L. Anchordoqui and H. Goldberg, arXiv:0709.0678] [hep-ph]. A. B. Balantekin and K. O. Ozansoy, arXiv:0710.0028 [hep-ph].

[7] E. Accomando et al. [CLIC Physics Working Group], arXiv:hep-ph/0412251. T. Abe et al. [American Linear Collider Working Group], in Proc. of the APS/DPF/DPB Summer Study on the Future of Particle Physics (Snowmass 2001) ed. N. Graf, arXiv:hep-ex/0106055. T. Abe et al. [American Linear Collider Working Group], in Proc. of the APS/DPF/DPB Summer Study on the Future of Particle Physics (Snowmass 2001) ed. N. Graf, 
arXiv:hep-ex/0106056. T. Abe et al. [American Linear Collider Working Group], in Proc. of the APS/DPF/DPB Summer Study on the Future of Particle Physics (Snowmass 2001) ed. N. Graf, arXiv:hep-ex/0106057. T. Abe et al. [American Linear Collider Working Group], in Proc. of the APS/DPF/DPB Summer Study on the Future of Particle Physics (Snowmass 2001) ed. N. Graf, arXiv:hep-ex/0106058. J. A. Aguilar-Saavedra et al. [ECFA/DESY LC Physics Working Group], arXiv:hep-ph/0106315. R. W. Assmann et al., A 3-TeV $e^{+} e^{-}$linear collider based on CLIC technology, CERN-2000-008, Geneva, 2000. R. W. Assmann et al., CLIC contribution to the technical review committee on a $500 \mathrm{GeV} e^{+} e^{-}$linear collider, CERN-2003-007, Geneva, 2003. A. De Roeck, arXiv:hep-ph/0311138.

[8] I. F. Ginzburg et al., Nucl. Instrum. Methods Phys. Res. 205. 47(1983); I. F. Ginzburg et al., Nucl. Instrum. Methods Phys. Res. A 219. 5(1984)

[9] S. Dawson and M. Oreglia, Ann. Rev. Nucl. Part. Sci. 54, 269 (2004) arXiv:hep-ph/0403015].

[10] H. Davoudiasl, Phys. Rev. D 61, 044018 (2000) [arXiv:hep-ph/9907347].

[11] O. Cakir, E. Ateser and H. Koru, Eur. Phys. J. C 28, 381 (2003); Z. Kirca, O. Cakir and Z. Z. Aydin, Acta Phys. Polon. B 34, 4079 (2003);

[12] W. M. Yao et al. [Particle Data Group], J. Phys. G 33, 1 (2006). 